\title{
55 \\ Hybrid interpretation of induction results
}

\author{
Xindong $W u$ \\ Department of Software Development, Monash University \\ 900 Dandenong Road, Melbourne, VIC 3145, Australia \\ Email: xindong@insect.sd.monash.edu.au
}

\begin{abstract}
When applying rules produced by induction from training examples to a test example, there are three possible cases that demand different actions: (1) no match; (2) single match; and (3) multiple match. Existing techniques for dealing with the first and third cases are exclusively based on probability estimation. However, when there are continuous attributes in the example space, and if these attributes have been discretized into intervals before induction, fuzzy interpretation of the discretized intervals at deduction time could be very valuable. This paper describes the fuzzy matching techniques implemented in the HCV (Version 2.0) software, and presents a hybrid interpretation mechanism which combines fuzzy matching with probability estimation. Experiment results of the HCV (Version 2.0) software with different interpretation techniques are provided on a number of data sets from the University of California at Irvine Repository of Machine Learning Databases.
\end{abstract}

\section{Keywords}

No match, multiple match, discretization of real-valued domains, fuzzy matching.

\section{INTRODUCTION}

Deduction and induction are two opposite operations in the area of knowledge discovery in databases (KDD). Induction generates knowledge in the form of, say, rules or decision trees from existing data and deduction applies induction results to interpret new data. Automatic knowledge acquisition from databases [ $\mathrm{Wu} 95$ ] includes three interconnected phases:

1. Translation of standard database information into a form suitable for use by machine learning facilities; 
2. Using rule induction techniques to produce knowledge bases from databases; and

3. Interpreting the knowledge produced to solve users' problems and reduce data spaces.

Since real world databases are normally incomplete and noisy, induction results from the 2 nd phase cannot be assumed to be perfect. When induction results take the form of rules, interpreting them to classify a new example in the 3rd phase needs to face three possible cases which demand different actions:

No match: No conjunctive rules match the example;

- Single match: One or more rules indicate the same class match; and

- Multiple match: More than one rule matches the example, and indicates different classes.

The third case does not apply to decision trees produced by ID3-like algorithms, but when the trees are decompiled into production rules [Quinlan 87, Quinlan 93], the production rules will face the same problems.

In the single match case, the choice of class to the example is naturally the class indicated by the rules. Deduction-time processing deals mainly with the conflict resolution in the third case and probability estimation for the first case. Existing techniques for dealing with the first and third cases are both exclusively based on probability estimation. Among them, the Measure of Fit for dealing with the no match case and the Estimate of Probability for handling the multiple match case developed in [Michalski et al. 86] have been widely adopted in the KDD community.

The Measure of Fit and Estimate of Probability methods perform quite well with problem domains where no real-valued attributes are involved. However, when a problem contains attributes that take values from continuous domains (i.e. real numbers or integers), their performance, especially in terms of accuracy, decreases. In existing induction algorithms, dealing with continuous domains is based on discretization of them into a certain number of intervals. There are quite a few strategies available for discretization, such as [Wu 95, Wu 96, Dougherty et al. 95, Quinlan 93, Fayyad \& Irani 92, Catlett 91] Bayesian discretizers and the information gain method. Once each continuous domain has been discretized into intervals, the intervals are treated as discrete values in induction and deduction. This is the standard way all existing induction systems have taken. However, discretization of continuous domains does not always fit accurate interpretation. To say an age greater than 50 is old or a temperature above 32 centigrades is high is fuzzy. In these kinds of cases, fuzzy interpretation of the discretized intervals at deduction time could be very valuable. Rather than taking the cut points decided by a discretization method as sharp borders for intervals, we can instead place some kind of curve at each cut point as fuzzy borders. With these fuzzy borders, a value can be classified into a few different intervals at the same time, with varying degrees. This could change a single match case to a multiple match, and a no match case to a single or even multiple match. Deduction with fuzzy borders of discretized intervals is called fuzzy matching. In the multiple match case, we can take the interval with the greatest degree as the value's discrete value.

This paper describes the fuzzy matching techniques implemented in the HCV (Version 2.0) software [Wu 95], and presents a hybrid interpretation mechanism which combines fuzzy matching with probability estimation. In the following section, we outline existing probability estimation techniques. In Section 3, we describe the fuzzy matching techniques 
developed and implemented in HCV (Version 2.0), and we follow this with the design of a hybrid interpretation mechanism in Section 4 which combines fuzzy matching with probability estimation. In Section 5, experiment results of the HCV (Version 2.0) software with different interpretation techniques are provided on a number of data sets from the University of California at Irvine Repository of Machine Learning Databases.

\section{PROBABILITY ESTIMATION IN THE CASES OF NO MATCH AND MULTIPLE MATCH}

\section{$2.1 \quad$ No match}

In the case of no match, we need to examine the training set and find a class which is close to the test example in question.

- Largest class. A common method to deal with no match is to assign all the no match examples to the largest class, called the default class [Clark \& Niblett 89]. The rationale behind this method is that if the examples in the training set are representative, the possibility of a random example belonging to a large class is higher than that it belongs to a small one.

The largest class method is good when the number of classes in a training set is small and one of the classes contains a predominant number of examples. However, the results deteriorate when the number of classes grows, and the number of examples in every class is more evenly spread out.

- Measure of fit. Rather than relying solely on the probability of each class in the training set, the Measure of Fit method [Michalski et al. 86] calculates the $M F$ value (Measure of Fit) of each class $c_{i}$ for each no match example $e$.

For a selector sel, $X=\left[V_{1}, \ldots, V_{n}\right]$, its $M F$ value for $e$ is defined as

$M F(s e l, e)= \begin{cases}1 & \text { if } \text { sel is satisfied by } e \\ \frac{n}{|X|} & \text { otherwise }\end{cases}$

where $|X|$ is the number of values in the $X$ domain.

The $M F$-value of a conjunctive rule conj is defined on the product of the $M F$-value of its selectors, adjusted by the rule's weight in the training set:

$M F(\operatorname{conj}, e)=\prod_{k} M F\left(\operatorname{sel}_{k}, e\right) \times \frac{n(\operatorname{conj})}{N}$

where $n$ (conj) is number of examples in the training set that are covered by the conj rule, and $N$ is the total number of examples in the training set.

The MF of a class $c_{i}$ is the probabilistic sum of all the conjunctive rules for the class. In the case of two rules, $c o n j_{1}$ and $c o n j_{2}$, it is given by the following formula

$$
M F\left(c_{i}, e\right)=M F\left(\operatorname{conj}_{1}, e\right)+M F\left(\operatorname{conj}_{2}\right)-M F\left(\operatorname{conj}_{1}, e\right) M F\left(\operatorname{conj}_{2}, e\right) .
$$


If there are more than two rules for the class, we use the above formula recursively.

The Measure of Fit method interprets the $M F$ value as the closeness of the example to the class, and chooses the class $c_{i}$ which maximize $M F\left(c_{i}, e\right)$ as $e$ 's classification.

\subsection{Multiple match}

Multiple match is caused by over-generalization of the training examples at induction time. All rule induction algorithms implement generalization and specialization, explicitly or implicitly. We try to generalize the positive examples as far as we can until it covers negative examples. Once negative examples are covered, we need to specialize the concept description to exclude them. However, since the training set is generally incomplete, we need to carry out generalization and specialization under the closed world assumption. A missing example, say $\left(X_{1}=a, X_{2}=1\right)$, can be assumed to belong to a concept $c$ when we have found no existing negative examples of the concept take the value of $a$ on $X_{1}$. It can also be assumed to be a negative example at the same induction process if we find that all the negative examples and no positive examples in the training set have the property of $X_{2}=1$. The example in this case could well be covered by the descriptions of both the concept and another concept (which might be 'not $c$ '), and therefore a multiple match happens in the subsequent deduction when the example appears in the test set.

Multiple match resolution needs to provide some measurements or criteria to judge which class is closer or more reliable to the test example in question.

- First hit. The simplest way to solve the multiple match issue is to use the first rule which classifies the example to determine the example's classification. If the rules from induction have been sorted and ordered according to their reliability or their class reliability (e.g. putting rules related to the largest class before others), this simple method can be expected to produce reasonable results.

The advantage of this method is that it is straightforward and efficient in execution time. However, the price for the efficience at deduction time is that the rules from induction need to be sorted.

- Largest class. The largest class method to solve multiple match examples has the same rationale as it is applied to the no match case. It counts the coverage of those classes in the training set which are applicable to the test example in question, and then selects the largest one.

This method can sometimes produce good results, but since it does not use any information about the structure of the problem domain, it is not always reliable.

- Largest rule. Instead of using the coverage of the rules for a class, we can use the coverage of each conjunctive rule. However, the analysis with the largest class method also applies to this method.

- Estimate of probability. The Estimate of Probability [Michalski et al. 86] for handling the multiple match case assigns an $E P$ value to every class by examing the size of the satisfied conjunctive rules. 
The $E P$ value for a conjunctive rule conj that is satisfied by an example $e$ is defined as

$E P(\operatorname{conj}, e)= \begin{cases}\frac{n(c o n j)}{N} & \text { if } \operatorname{con} j \text { is satisfied by } e \\ 0 & \text { otherwise }\end{cases}$

where $n(c o n j)$ is the weight of $c o n j$ and $N$ is the number of examples in the training set.

The EP value of a class $c_{i}$ is defined as the probabilistic sum of all the conjunctive rules for the class. In the case of two rules, $c o n j_{1}$ and $c o n j_{2}$, it is given by the following formula

$E P\left(c_{i}, e\right)=E P\left(\operatorname{conj}_{1}, e\right)+E P\left(\operatorname{conj}_{2}\right)-E P\left(\operatorname{conj}_{1}, e\right) E P\left(\operatorname{conj}_{2}, e\right)$

If there are more than two rules for the class, we use the above formula recursively.

The Estimate of Probability method chooses the class with the highest $E P$ value to classify the example.

In Section 5, the largest class method for both no match and multiple match cases, and a combination of the Estimate of Probability (for multiple match) with fuzzy techniques described in Section 4, called hybrid, will be used in the experiments.

\section{$3 \quad$ FUZZY MATCHING IN HCV (VERSION 2.0)}

\subsection{The HCV (Version 2.0) Software}

The HCV algorithm [Wu 93] is an attribute-based induction algorithm based on the newly developed extension matrix approach originated with J.R. Hong's AE1 [Hong 85]. By dividing the positive examples (PE) of a specific concept in a given example set into intersecting groups and adopting a set of strategies to find a heuristic conjunctive rule in each group which covers all the group's positive examples and none of the negative examples (NE), HCV can find a rule in the form of variable-valued logic [Michalski 75] for the concept in low-order polynomial time. If there exists at least one conjunctive rule in a given training example set for PE against NE, the rule produced by HCV must be a conjunctive one.

The HCV (Version 2.0) Software is a $\mathrm{C}++$ implementation of the HCV algorithm. In this implementation, HCV can work with noisy and real-valued domains as well as nominal and noise-free databases. It also provides a set of deduction facilities for the user to test the accuracy of the produced rules on test examples. The detailed description of the software is included in [Wu 95]. Below we outline the fuzzy matching techniques designed for dealing with real-valued attributes at deduction time.

\subsection{Fuzzy Interpretation of Induction Results}

Rather than taking the cut points set up by a discretization method as sharp borders, each interval is associated with a specific membership function with fuzzy methods. The membership function measures the degree of a value belonging to the interval. In fact, 
sharp intervals can be treated as a special case of fuzzy borders: the membership function for an interval with sharp borders takes value 1 iff the value is inside the interval and 0 otherwise, and one value can belong to one interval only. Figure 1 shows the difference between sharp borders and fuzzy ones.

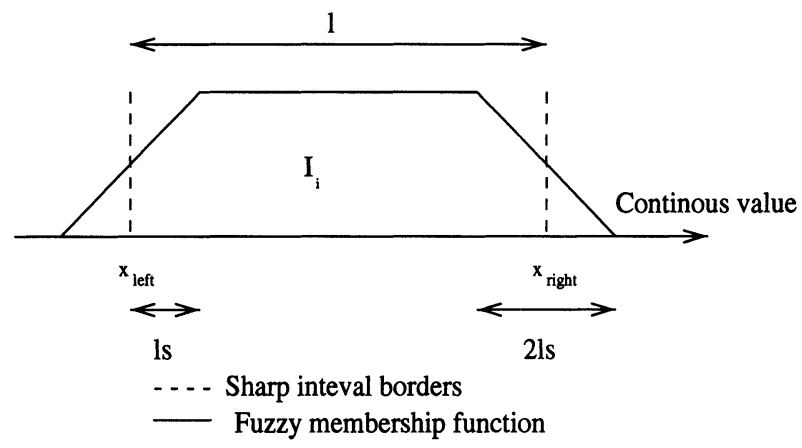

Figure 1 Sharp and Fuzzy Borders

In HCV (Version 2.0), $s$ is a user-specified parameter with default being 0.1 , which indicates the length that an interval should be extended at each end. If the parameter is 0.1, the interval in Figure 1 spreads out into adjacent intervals for twenty percent of its original length.

There are three different functions which are available for use in HCV (Version 2.0) to fuzzify the borders. In the following account of these functions, $s$ is the spread parameter as described above, $x_{l e f t}$ and $x_{\text {right }}$ are the left and right sharp borders respectively (determined by an information gain based discretization method [Wu et al. 95]), and $l=x_{\text {right }}-x_{\text {left }}$ is the original length of the interval.

The linear function is specified by the following expressions.

$$
\begin{gathered}
k=\frac{1}{2 s l}, \quad a=-k x_{\text {left }}+\frac{1}{2}, \quad b=k x_{\text {right }}+\frac{1}{2} \\
\operatorname{lin}_{\text {left }}(x)=k x+a \\
\operatorname{lin}_{\text {right }}(x)=-k x+b \\
\operatorname{lin}(x)=M A X\left(0, M I N\left(1, \operatorname{lin}_{\text {left }}(x), \operatorname{lin}_{\text {right }}(x)\right)\right)
\end{gathered}
$$

With the polynomial membership function, the fuzzy borders are defined by a thirddegree polynomial.

where

$$
\begin{gathered}
\operatorname{poly}_{\text {left }}(x)=a_{\text {left }} x^{3}+b_{\text {left }} x^{2}+c_{\text {left }} x+d_{\text {left }} \\
\text { poly } y_{\text {right }}(x)=a_{\text {right }} x^{3}+b_{\text {right }} x^{2}+c_{\text {right }} x+d_{\text {right }}
\end{gathered}
$$

$$
\begin{gathered}
a_{l e f t}=a_{r i g h t}=-\frac{1}{4(l s)^{3}} \\
b_{s i d e}=-3 a_{\text {side }} x_{s i d e} \\
c_{\text {side }}=3 a_{\text {side }}\left(x_{\text {side }}^{2}-(l s)^{2}\right)
\end{gathered}
$$




$$
d_{\text {side }}=-a\left(x_{\text {side }}^{3}-3 x_{\text {side }}(l s)^{2}+2(l s)^{3}\right)
$$

and side $\in\{$ left,right $\}$ and $x_{\text {side }}$ is the sharp border on each side.

$$
\operatorname{poly}(x)= \begin{cases}\text { poly } y_{\text {left }}(x) & \text { if } x_{\text {left }}-l s \leq x \leq x_{\text {left }}+l s \\ \text { poly } y_{\text {right }}(x) & \text { if } x_{\text {right }}-l s \leq x \leq x_{\text {right }}+l s \\ 1 & \text { if } x_{\text {left }}+l s \leq x \leq x_{\text {right }}-l s \\ 0 & \text { otherwise }\end{cases}
$$

The third membership function is the arctan function. The spread of the interval is used to indicate the flatness or linearity of the curve, and the fuzzy membership of an interval takes the minimum of the membership from the left and the one from the right. The function used to calculate the membership is:

$$
\arctan (x)=\operatorname{MIN}\left(\frac{1}{\pi} \operatorname{atan}\left(\frac{x-x_{\text {left }}}{5 s}\right)+\frac{1}{2}, \frac{-1}{\pi} \operatorname{atan}\left(\frac{x-x_{\text {right }}}{5 s}\right)+\frac{1}{2}\right) .
$$

The match of an example taking value $x$ on a specific attribute domain with an interval is defined as the value of the membership function of the interval calculated for $x$.

With all of the three fuzzy membership functions, two methods for calculating the match degree of a value $x$ with a selector or conjunction have been implemented. The first takes the maximum membership degree of the value in all of the intervals involved in the selector. The drawback of this method is that if two adjacent intervals belong to the same selector, a value close to the border between the two intervals will get a very low membership value in both, leading to a low overall membership degree even if it is well covered by the selector. In an attempt to remedy this, the other method adds with fuzzy plus* all the fuzzy membership degrees within one selector.

\section{HYBRID INTERPRETATION}

Extensive experiments have been carried out with the above fuzzy methods on a large set of databases from the University of California at Irvine Repository of Machine Learning Databases. However, the results were much less encouraging than what we expected when we were trying to justify that fuzzy borders are generally more reliable than sharp borders with numerical domains.

We have analysed the results by fuzzy methods and those with sharp borders, and found that the accuracy of the single matches is in general much better than no matches and multiple matches with all methods. With the multiple match case, the Estimate of Probability [Michalski et al. 86] with the Laplacian Law of Succession ${ }^{\dagger}$ [Niblett and Bratko 87]

\footnotetext{
*Fuzzy plus $\oplus$ is defined as follows: $a \oplus b=a+b-a b$.

†In formula (3) of the original Estimate of Probability method, probability estimation is based on the frequency method. Given that an event has occurred $n$ times out of $N$ attempts, the frequency method for estimating the probability of $e, p(e)$, is to use its relative frequency, $n / N$. With the Laplacian Law of Succession [Niblett and Bratko 87], the following formula is used to estimate the probability of an event $e$ under the same assumption as the frequency method
}

$p(e)=\frac{n+1}{N+2}$ 
outperforms other methods including fuzzy matching. These observations motivated the development of a hybrid interpretation in HCV (Version 2.0) with fuzzy matching and the Estimate of Probability.

The hybrid method works as follows. In the single match case, we do not provide any probability analysis or fuzzy borders. In the multiple match case, the Estimate of Probability method with sharp borders is used to find the best class for the example in question. Only in the no match case, fuzzy borders are set up (with the polynomial membership function as default) in order to find a rule which is closest (with the maximum membership degree) to the example in question.

The hybrid method has been chosen as the default method for deduction in HCV (Version 2.0). However, the user can overrule it by specifying other methods (such as the combination of the Measure of Fit and the Estimate of Probability).

\section{EXPERIMENT RESULTS}

Table 1 shows accuracy results by HCV (Version 2.0) with different deduction techniques on 17 different data sets, all of which contain noise and continuous attributes. These data sets are all available from the University of California at Irvine Repository of Machine Learning Databases. HCV (hybrid) in Table 1 indicates the hybrid interpretation approach designed in Section 4. HCV (large) refers to the largest class method mentioned in Section 2 for both no match and multiple match cases. HCV (fuzzy) refers to the fuzzy matching techniques and default parameters designed in Section 3.2, with the polynomial membership function as default. In addition to HCV (Version 2.0) with these different deduction strategies, we have also chosen C4.5 [Quinlan 93] and NewID [Boswell 90] from the ID3 [Quinlan 86] like algorithms to compete with HCV (Version 2.0) in this section. Both C4.5 and NewID have provided facilities to deal with real-valued and nominal attributes. C4.5 is the most recent successor of ID3-like algorithms. NewID does binarization of decision trees, which means each non-leaf node has at most two child nodes.

Apart from the deduction strategy mentioned above for HCV (Version 2.0), the results were produced by using these programs' default parameters. The results shown for C4.5 and NewID are the pruned ones. The best result for each problem is highlighted with boldface font in the table.

Of the 17 different problems, C4.5 has the best results for 7, NewID for 3, and HCV (hybrid) gets the best results for 9 . The sum is greater than 17 because sometimes different algorithms get the same results for some problems.

¿From Table 1, the fuzzy matching techniques generally perform better than the simple largest class method. However, the performance of HCV (Version 2.0) improves significantly when fuzzy matching is combined with probability estimation. For example, HCV (fuzzy) performs worse than C4.5 on 11 example sets and better on only 4, whereby HCV (hybrid) performs worse than C4.5 on only 7 example sets but better on 9 .

where $n$ and $N$ have the same meanings as in the frequency method. 
Table 1 Accuracy Comparison with Continuous Domains

\begin{tabular}{cccccc}
\hline Domain & HCV (hybrid) & C4.5 & NewID & HCV (large) & HCV (fuzzy) \\
\hline Anneal & $\mathbf{9 8 . 0 \%}$ & $93.0 \%$ & $81.0 \%$ & $93.0 \%$ & $93.0 \%$ \\
Bupa & $57.6 \%$ & $61.0 \%$ & $\mathbf{7 3 . 0 \%}$ & $55.9 \%$ & $55.9 \%$ \\
Cleveland 2 & $\mathbf{7 8 . 0 \%}$ & $76.9 \%$ & $67.0 \%$ & $68.1 \%$ & $73.6 \%$ \\
Cleveland 5 & $54.9 \%$ & $\mathbf{5 6 . 0 \%}$ & $47.3 \%$ & $\mathbf{5 6 . 0 \%}$ & $52.7 \%$ \\
Crx & $\mathbf{8 2 . 5 \%}$ & $80.0 \%$ & $79.0 \%$ & $72.5 \%$ & $82.0 \%$ \\
Glass (without ID number) & $\mathbf{7 2 . 3 \%}$ & $64.6 \%$ & $66.0 \%$ & $60.0 \%$ & $60.0 \%$ \\
Hungarian 2 & $\mathbf{8 6 . 3 \%}$ & $80.0 \%$ & $78.0 \%$ & $85.0 \%$ & $85.0 \%$ \\
Hypothyroid & $97.8 \%$ & $\mathbf{9 9 . 4 \%}$ & $92.0 \%$ & $86.3 \%$ & $96.3 \%$ \\
Imports 85 & $62.7 \%$ & $\mathbf{6 7 . 8 \%}$ & $61.0 \%$ & $59.3 \%$ & $61.0 \%$ \\
Ionosphere & $\mathbf{8 8 . 0 \%}$ & $85.5 \%$ & $82.0 \%$ & $81.2 \%$ & $81.2 \%$ \\
Labor Neg & $76.5 \%$ & $\mathbf{8 2 . 4 \%}$ & $65.0 \%$ & $76.5 \%$ & $76.5 \%$ \\
Pima & $73.9 \%$ & $\mathbf{7 5 . 5 \%}$ & $73.0 \%$ & $69.1 \%$ & $69.1 \%$ \\
Swiss 2 & $\mathbf{9 6 . 9 \%}$ & $\mathbf{9 6 . 9 \%}$ & $\mathbf{9 7 . 0 \%}$ & $\mathbf{9 6 . 9 \%}$ & $\mathbf{9 6 . 9 \%}$ \\
Swiss 5 & $28.1 \%$ & $\mathbf{3 1 . 2 \%}$ & $22.0 \%$ & $25.0 \%$ & $28.1 \%$ \\
Va 2 & $\mathbf{7 8 . 9 \%}$ & $70.4 \%$ & $77.0 \%$ & $\mathbf{7 8 . 9 \%}$ & $\mathbf{7 8 . 9 \%}$ \\
Va 5 & $28.2 \%$ & $26.8 \%$ & $20.0 \%$ & $25.4 \%$ & $\mathbf{2 9 . 6 \%}$ \\
Wine & $\mathbf{9 0 . 4 \%}$ & $90.0 \%$ & $\mathbf{9 0 . 4 \%}$ & $76.9 \%$ & $76.9 \%$ \\
\hline
\end{tabular}

\section{CONCLUSIONS}

As mentioned in Section 4, fuzzy methods, although their results are significant when combined with other deduction methods, do not contribute as much as one can expect to the accuracy of deduction on their own. This is likely because all the experiments have not been specifically conducted with domain dependent information. Fuzziness is strongly domain dependent. The HCV (Version 2.0) has provided a way for the user to specify their own intervals and select their own fuzzy functions. This is an important direction to take if we would like to achieve significant results with specific domains.

This paper has introduced the fuzzy matching techniques implemented in the HCV (Version 2.0) software, and a hybrid interpretation mechanism which combines fuzzy matching with probability estimation. Experiment results of the HCV (Version 2.0) software with different interpretation techniques have shown that the hybrid interpretation can achieve much better results in terms of predictive accuracy than fuzzy matching alone.

\section{REFERENCES}

[Boswell 90] Boswell, R. (1990) Manual for NewID Version 6.1. TI/P2154/RAB/4/2.5, The Turing Institute, Glasgow.

[Catlett 91] Catlett, J. (1991) On Changing Continuous Attributes into Ordered Discrete 
Attributes, in Proceedings of the 1991 European Working Session on Learning.

[Clark \& Niblett 89] Clark, P. and Niblett, T. (1989) The CN2 Induction Algorithm. Machine Learning, 3, 261-283.

[Dougherty et al. 95] Dougherty, J., Kohavi, R. and Sahami, M. (1995) Supervised and Unsupervised Discretization of Continuous Features, in Proceedings of the 12th International Conference on Machine Learning, 194-202.

[Fayyad \& Irani 92] Fayyad, U.M. and Irani, K.B. (1992) On the Handling of ContinuousValued Attributes in Decision Tree Generation. Machine Learning, 8, 87-102.

[Hong 85] Hong, J. (1985) AE1: An Extension Matrix Approximate Method for the General Covering Problem. International Journal of Computer and Information Sciences, 14, 6: 421-437.

[Michalski 75] Michalski, R.S. (1975) Variable-Valued Logic and Its Applications to Pattern Recognition and Machine Learning, in Computer Science and Multiple-Valued Logic Theory and Applications, (ed. D.C. Rine), Amsterdam: North-Holland, 506-534.

[Michalski et al. 86] Michalski, R.S., Mozetic, I., Hong, J. and Lavrac, N. (1986) The MultiPurpose Incremental Learning System AQ15 and Its Testing Application to Three Medical Domains, in Proceedings of AAAI 1986, 1041-1045.

[Niblett and Bratko 87] Niblett, T. and Bratko, I. (1987) Learning Decision Rules in Noisy Domains, in Research and Development in Expert Systems III (ed. M.A Bramer), Cambridge University Press, Cambridge, 25-34.

[Quinlan 86] Quinlan, J.R. (1986) Induction of Decision Trees. Machine Learning, 1, 81106.

[Quinlan 87] Quinlan, J.R. (1987) Generating Production Rules from Decision Trees, in Proceedings of International Joint Conference on Artificial Intelligence (ed. J. McDermott), Morgan Kaufmann Publishers, Inc., 304-307.

[Quinlan 93] Quinlan, J.R. (1992) C4.5: Programs for Machine Learning. Morgan Kaufmann Publishers.

[Wu 93] Wu, X. (1993) The HCV Induction Algorithm, in Proceedings of the 21st ACM Computer Science Conference (eds. S.C. Kwasny and J.F. Buck), ACM Press, USA, $168-175$.

[Wu 95] Wu, X. (1995) Knowledge Acquisition from Data Bases. Ablex Publishing Corp., U.S.A..

[Wu 96] Wu, X. (1996) A Bayesian Discretizer for Real-Valued Attributes. Technical Report 96-1, Dept. of Software Development, Monash University, Melbourne.

[Wu et al. 95] Wu, X., Krisár, J. and Måhlén, P. (1995) Noise Handling with Extension Matrices, in Proceedings of the 7th IEEE International Conference on Tools With Artifcial Intelligence, Hyatt Dulles, Washington, D.C., USA, November 5-8, IEEE Computer Society Press, 190-197. 